\title{
Utilizing Mind-Maps for Information Retrieval and User Modelling
}

\author{
Joeran Beel ${ }^{1,2}$, Stefan Langer ${ }^{1,2}$, Marcel Genzmehr ${ }^{1}$, Bela Gipp ${ }^{1,3}$ \\ ${ }^{1}$ Docear, Magdeburg, Germany \\ ${ }^{2}$ Otto-von-Guericke University, Magdeburg, Germany \\ ${ }^{3}$ University of California, Berkeley, USA \\ \{beel | langer | genzmehr | gipp\}@docear.org
}

\begin{abstract}
Mind-maps have been widely neglected by the information retrieval (IR) community. However, there are an estimated two million active mind-map users, who create 5 million mind-maps every year, of which a total of 300,000 is publicly available. We believe this to be a rich source for information retrieval applications, and present eight ideas on how mind-maps could be utilized by them. For instance, mind-maps could be utilized to generate user models for recommender systems or expert search, or to calculate relatedness of web-pages that are linked in mind-maps. We evaluated the feasibility of the eight ideas, based on estimates of the number of available mind-maps, an analysis of the content of mind-maps, and an evaluation of the users' acceptance of the ideas. We concluded that user modelling is the most promising application with respect to mind-maps. A user modelling prototype - a recommender system for the users of our mind-mapping software Docear - was implemented, and evaluated. Depending on the applied user modelling approaches, the effectiveness, i.e. click-through rate on recommendations, varied between $0.28 \%$ and $6.24 \%$. This indicates that mind-map based user modelling is promising, but not trivial, and that further research is required to increase effectiveness.
\end{abstract}

Keywords: mind-maps, content analysis, user modelling, information retrieval

\section{Introduction}

Information retrieval (IR) applications utilize many items beyond the items' original purpose. For instance, emails are intended as a means of communication, but Google utilizes them for generating user profiles and displaying personalized advertisement [1]; social tags can help to organize private web-page collections, but search engines utilize them for indexing websites [2]; research articles are meant to publish research results, but they, or more precisely their references, are utilized to analyze the impact of researchers and institutions [3].

We propose that mind-maps are an equally valuable source for information retrieval as are social tags, emails, research articles, etc. Consequently, our research objective was to identify, how mind-maps could be used to empower IR applications. To 
achieve our objective, we 1) analyzed the extent to which mind-mapping is used, to decide if mind-map based IR is a field worth researching, 2) brainstormed how mindmaps might be utilized by IR applications, 3) analyzed the feasibility of the ideas, and 4) implemented a prototype of the most promising idea, which - to anticipate the result - is a recommender system that creates user models based on mind-maps. All estimates in this paper are based on data collected from our own mind-mapping software Docear [4, 5], Google Trends and the mind-mapping tools' websites.

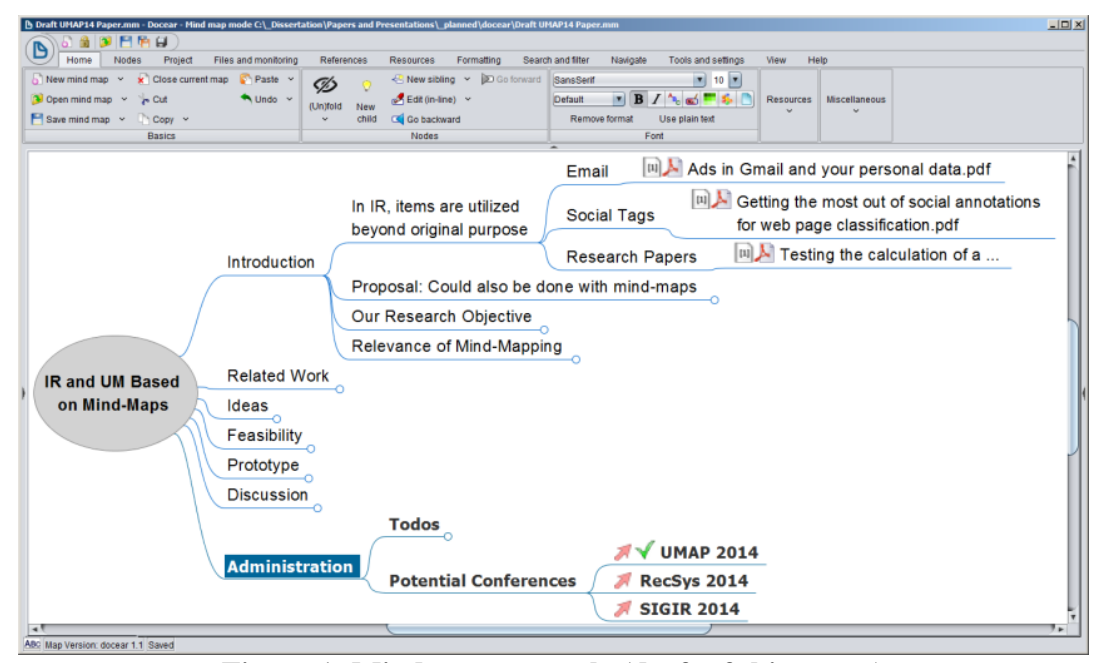

Figure 1: Mind-map example (draft of this paper)

We hope to stimulate a discussion with this paper that encourages IR and user modelling researchers to further analyze the potential of mind-maps. We believe that researchers will find this new research field rewarding, and the results will enable developers of mind-mapping tools to devise novel services for their millions of users.

\section{Related Work}

Mind-maps are typically used to develop ideas and organize information. As such they are often used for tasks including brainstorming, project management and document drafting. Figure 1 shows an example of a mind-map, created with our mindmapping software Docear (http://docear.org) [5]. We created the mind-map to represent a draft of this paper. The root node represents the title of this paper. From the root node, child nodes branch to represent each chapter, additional child nodes branch off for each paragraph, sentence and reference. We also added a list of relevant conferences, to which we planned to submit the paper. Red arrows indicate a link to a website. A PDF icon indicates a link to a PDF file on the hard drive. A "circle" on a node indicates that the node has child nodes that are currently hidden.

There has been plenty of research showing the effectiveness of mind-mapping as a learning tool [6]; creating mind-maps automatically from full-text streams [7]; and evaluating whether paper-based or electronic mind-mapping is more effective [8]. To the best of our knowledge, mind-maps have not been researched with regard to infor- 
mation retrieval or user modelling. However, there are two types of information retrieval applications, which utilized mind-maps in practice.

The first type of application is a search engine for mind-maps. Several mindmapping tools, for instance XMind and MindMeister, allow their users to publish their mind-maps in so called "mind-map galleries". These galleries are similar to photo galleries. They show thumbnails of mind-maps that users uploaded to the gallery. Visitors of the galleries may search for mind-maps containing certain keywords, and download the corresponding mind-maps. According to MindMeister, around 10\% of mind-maps being created by their users are published in the galleries ${ }^{1}$. The other mind-maps remain private.

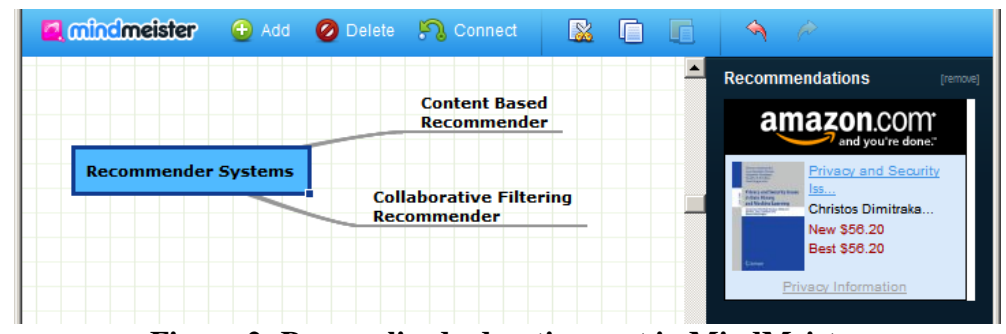

Figure 2: Personalized advertisement in MindMeister

The second type of application is a user modelling system. Only two companies MindMeister and Mindomo - implemented such a system to generate user models and display personalized advertisement. MindMeister extracted the terms of the node that a user last edited or created - typically, a node contains two or three terms [9]. These terms were sent to Amazon's Web Service as search query. Amazon returned book recommendations matching the search query, which MindMeister displayed in a window besides the mind-map (Figure 2). Mindomo had a similar concept, only that Google AdSense instead of Amazon was used. Meanwhile, both companies abandoned personalized advertisement, though they still offer and actively maintain their mind-mapping tools. In an email, Mindomo said that "people were not really interested" in the advertisement ${ }^{2}$.

\section{Popularity of Mind-Mapping and Mind-Mapping Tools}

Some reviewers of previous papers were skeptical whether there is enough interest in mind-mapping to justify the effort for researching the potential of IR applications utilizing mind-maps. We believe this skepticism to be unfounded, because, as shown in the next paragraphs, there is a significant number of mind-mapping tools and users who could benefit from the research.

The popularity of mind-mapping, based on search volume, is similar to the popularity of e.g. note taking, file management, or crowdsourcing, and significantly higher than for reference management, user modelling, recommender systems, or infor-

\footnotetext{
${ }^{1}$ Email from MindMeister's CEO Michael Hollauf, June 28, 2011. Permission for publication was granted.

${ }^{2}$ Email by Daniel Sima of the Mindomo team, October 3, 2011. Permission for publication was granted.
} 
mation retrieval (Figure 3). The website Mind-Mapping.org lists 142 mind-mapping tools being actively maintained, although some tools offer mind-mapping only as secondary feature in addition to other visualization techniques, such as concept maps or Gantt charts. When discontinued tools are included in the count, there are 207 tools. Of the 'pure' mind-mapping tools, i.e. those that focus on mind-mapping functionality, XMind is the most popular tool, based on search volume (25\%) (Figure 4$)^{3}$. Other popular tools are FreeMind (23\%), MindManager (13\%), and MindMeister $(8 \%)$. The search volume for XMind is in the same league as search volume for the Dropbox alternative ownCloud, the reference manager Zotero, or the Blog TechCrunch, and the volume is significantly higher than for academic conferences such as UMAP, SIGIR, or RecSys (Figure 5).

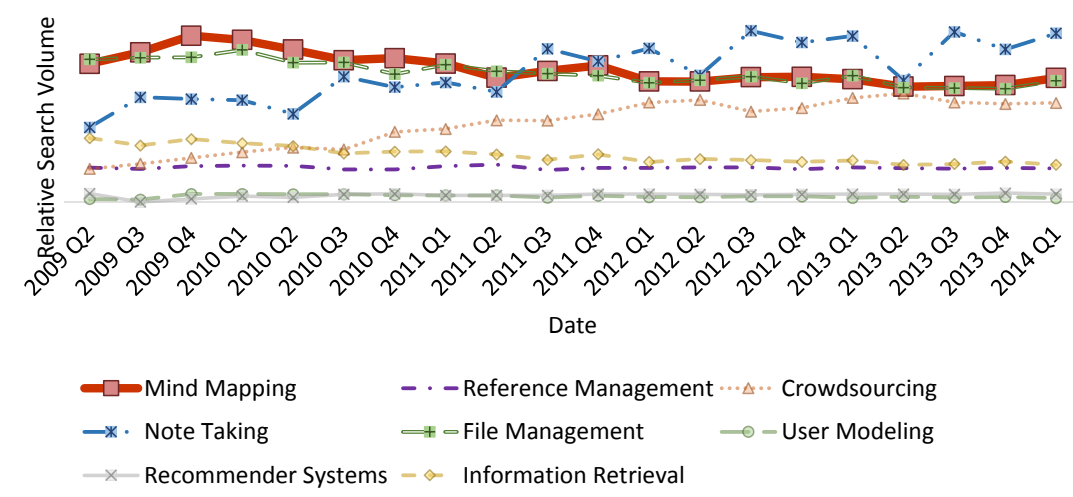

Figure 3: Search volume for "Mind-Mapping" and other selected search terms

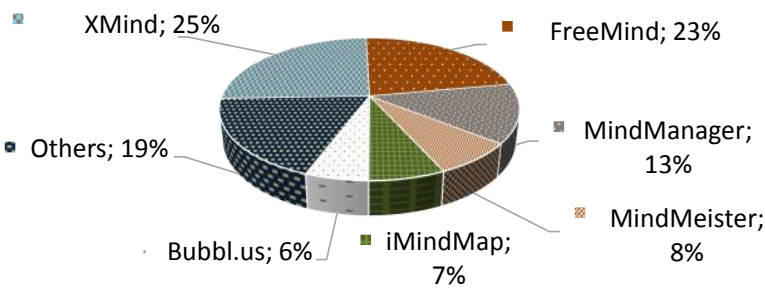

Figure 4: Search volume for mind-mapping tools

According to the tools' websites, XMind has more than 1 million users, Bubbl.us more than 1.5 million, MindManager more than 2 million, and MindMeister more than 2.5 million users. In sum, this makes 7 Million users for four tools that accumulate $52 \%$ of the search volume (Figure 4). Interpolating from the search volume, we can estimate that the remaining tools ( $48 \%$ of the search volume) must have around 6.5 million users. This results in a total of around 13.5 million mind-map users. To us, it seems likely that these numbers also include inactive users. For our own mindmapping software Docear, 10 to $20 \%$ of the users who registered in the past years, are

${ }^{3}$ All numbers relating to search volume are based on Google Trends http://www.google.com/trends/. Search volume is calculated relatively by Google, as such there are no numbers to display on the y-axis. 
active, i.e. they started Docear in the past month. Based on this information, we may estimate the numbers of active mind-map users to be between 1.35 and 2.7 million.

The claimed user counts do not always correlate with the search volume. For instance, MindMeister accumulates less than $8 \%$ of the search volume, and claims 2.5 million users. In contrast, XMind accumulates $25 \%$ of the search volume, but reports only around 1 million users. We assume that these differences result from different registration and usage concepts. MindMeister is a web-based tool that requires everyone to register. XMind is a desktop software that can also be used without registration. As such, our estimate remains a rough guess. However, another estimate leads to a similar result. The open source mind-mapping software FreeMind was downloaded 1.4 million times in the past 12 month (we considered only downloads of the latest stable release $)^{4}$. Assuming, that the number of active users is around $1 / 3$ of users who downloaded the software in the past year, leads to the estimate that FreeMind has around 450,000 active users. Interpolating from the search volume (22.58\%), leads to an estimate of 2 million active mind-map users.

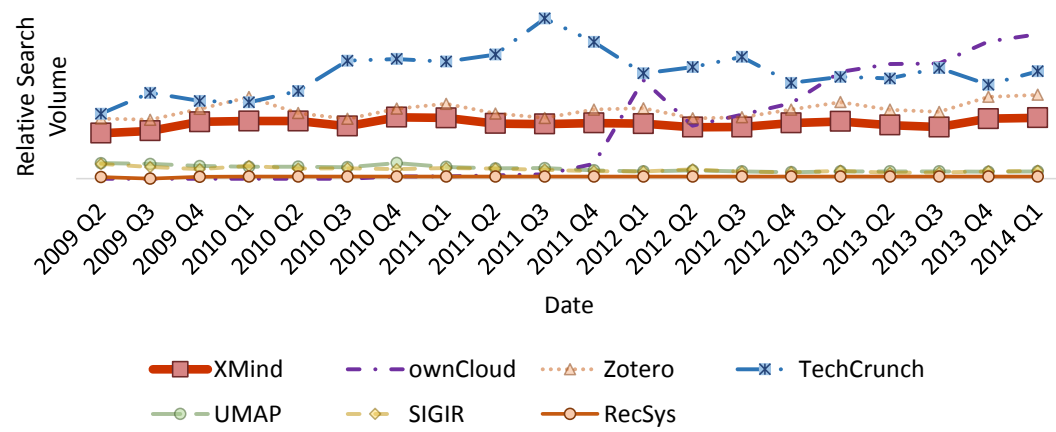

Figure 5: Search volume for "XMind" and other selected search terms

We believe that these numbers indicate a substantial interest in the topic of mindmapping, and the active user base justifies the effort to research the potential of utilizing mind-maps for IR applications.

\section{Ideas for Mind-Map based IR Applications}

To develop ideas, how IR applications could utilize mind-maps, we conducted a brainstorming session with a group of five experts from the fields of mind-mapping (two experts) and information retrieval (three experts). Before the session was conducted, the information retrieval experts were given an introduction to mind-mapping and the mind-mapping experts were given an introduction on how information retrieval is applied to emails, social tags, etc. The following eight ideas evolved from the brainstorming (for more details, refer to [10]).

Search Engines for Mind-Maps: Mind-maps contain information that probably is not only relevant for the given authors of a mind-map, but also for others. Therefore,

\footnotetext{
${ }^{4} \mathrm{http}: / /$ sourceforge.net/projects/freemind/files/stats/timeline
} 
a search engine for mind-maps might be an interesting application. As described in the "Related Work" section, such systems already exist.

User Modelling: Analog to analyzing users' authored research papers, emails, etc., user modelling systems could analyze mind-maps to identify users' information needs and expertise. User models could be used, for instance, for personalized advertisements, or by recommender systems, or expert search systems. For instance, when employees create mind-maps, we would assume that the mind-maps would be suitable to infer the employees' expertise. This information could be used by an expert search system. As described in the "Related Work" section, Mindomo and MindMeister implemented user modelling systems, but Mindomo reported that users were not interested in the results. Hence, they removed the system from their mind-mapping application. Apparently, user modelling based on mind-maps is not trivial and does not always lead to satisfying results.

Document Indexing / Anchor Text Analysis: Mind-maps could be seen as neighboring documents to those documents being linked in the mind-maps, and anchor text analysis could be applied to index the linked documents with the terms occurring in the mind-maps. Such information could be valuable, e.g., for classic search engines.

Document Relatedness: When mind-maps contain links to web pages or other documents, these links could be used to determine relatedness of the linked web pages or documents. For instance, with citation proximity analysis [11], documents would be assumed to be related that are linked in close proximity, e.g. in the same sentence. Such calculations could be relevant for search engines and recommender systems.

Document Summarization: Mind-maps could be utilized to complement document summarization. If a mind-map contains a link to a web-page, the node's text, and maybe the text of parent nodes, could be interpreted as a summary for the linked web page. Such summaries could be displayed by search engines on their result pages.

Impact Analysis: Mind-maps could be utilized to analyze the impact of the documents linked within the mind-map, similar to PageRank or citation based similarity metrics. This information could be used by search engines to rank, e.g., web pages, or by institutions to evaluate the impact of researchers and journals.

Trend Analysis: Trend analysis is important for marketing and customer relationship management, but also in other disciplines [12]. Such analyses could be done based on mind-maps. For instance, analyzing mind-maps that stand for drafts of academic papers would allow estimating citation counts for the referenced papers. It would also predict in which field new papers can be expected.

Semantic Analysis: A mind-map is a tree and nodes are in hierarchical order. As such, the nodes and their terms are in direct relationship to each other. These relationships could be used, for instance, by search engines to identify synonyms, or by recommender systems to recommend alternative search terms or social tags.

\section{$5 \quad$ Feasibility}

We evaluated the ideas' feasibility in three steps. First, we estimated whether there are enough mind-maps and mind-map users available to realize the ideas. Second, we analyzed whether the content of mind-maps is suitable for realizing the ideas. Finally, we gauged whether users are accepting the ideas. 


\subsection{Number of Mind-Map Users and (Public) Mind-Maps}

Most of the ideas hinge on the availability of a large number of mind-maps. It is also important to distinguish between public and private mind-maps. If many mind-maps were available publicly, the ideas could be realized by anyone. If mind-maps were private, i.e. only available to the developers of the mind-mapping tools, only these developers could realize the ideas.

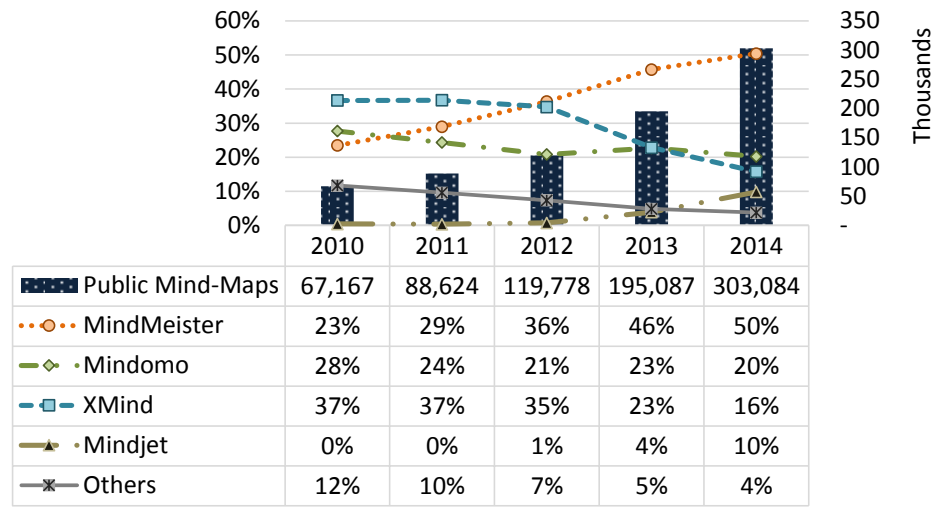

Figure 6: Public mind-maps

There are more than 300,000 mind-maps in public galleries, $50 \%$ of them in the gallery of MindMeister, 20\% in the gallery of Mindomo, and 16\% in the gallery of XMind (Figure 6) ${ }^{5}$. Over the years, the number of public mind-maps increased from 67,167 in 2010 to 303,084 in 2014. Given, that MindMeister's users published around 62,000 mind-maps between 2013 and 2014, we estimate that MindMeister's users created approximately 620,000 mind-maps during that period, since around $10 \%$ of mind-maps being created are also published ${ }^{1}$. Interpolating these numbers with the search volume (Figure 4), we can estimate that overall 4.6 million mind-maps were created between 2013 and 2014. Another estimate confirms this number: Mind-map users create between 2 and 3 mind-maps per year on average [9]. A calculation with 2.5 mind-maps per year, and 2 million mind-map users, leads to an estimate of 5 million mind-maps created per year. Considering that mind-mapping tools have been used for many years, a few dozens of millions mind-maps must exist on the computers of mind-map users.

\subsection{Content of Mind-Maps}

We recently analyzed the content of 19,379 mind-maps, created by 11,179 MindMeister and Docear users [9]. On average, mind-maps contained a few dozens of nodes, each with two to three words on average. Some mind-maps even contained a few thousand nodes, with some nodes containing more than a hundred words. This amount of nodes, and words, is comparable to the number of words in emails or web

\footnotetext{
${ }^{5}$ Over the past four years, we retrieved the numbers of mind-maps each year directly from the web-pages of the galleries.
} 
pages. Since emails and web pages are successfully utilized by information retrieval applications, the content of mind-maps might be suitable for those ideas that depend on the existence of terms. However, the number of links in mind-maps is low. Almost two thirds of the mind-maps did not contain any links to files, such as academic articles or other documents $(63.88 \%)$, and most of the mind-maps that did contain links, contained only few of them. Links to web-pages were not available in $92.37 \%$ of Docear's mind-maps and $75.27 \%$ of MindMeister's mind-maps. Consequently, those ideas based on link-analysis seem less attractive.

\subsection{User Acceptance}

We evaluated the user acceptance of the eight ideas with our mind-mapping software SciPlore MindMapping [13]. 4,332 users were shown at first start a settings dialog. In this dialog, users could (un)select four options relating to the different ideas we proposed (Figure 7). It was randomly chosen whether options were pre-selected.

When all options were pre-selected, $61 \%$ of the users accepted user modelling to receive recommendations based on their mind-maps (Figure 7). 38\% of the users accepted that the content of their mind-maps could be utilized e.g. for anchor text analysis. $32 \%$ of users agreed that SPLMM could also analyze the content of the documents they linked in their mind-maps. Usage mining, i.e. the general analysis of how users are making use of a software, was accepted by $48 \%$ of the users.

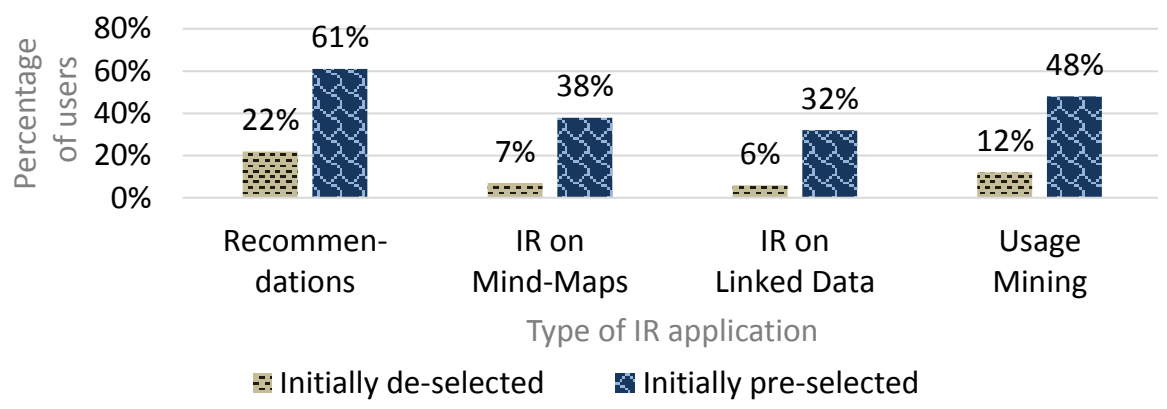

Figure 7: User acceptance of IR on their mind-maps

If options were not pre-selected, fewer users allowed the analysis of their data. $22 \%$ activated recommendations, $7 \%$ activated information retrieval on mind-maps, $6 \%$ activated IR on the linked documents, and $12 \%$ activated usage mining.

\subsection{Discussion of the Feasibility}

Due to the generally few links available in mind-maps, anchor text analysis, calculating document relatedness, document summarization, and impact analysis seem less feasible for the majority of mind-mapping tools (Table 1). However, there might be exceptions, for instance in the case of Docear. Docear's mind-maps contain comparatively many links to PDF files, because most users are researchers who manage their academic papers with Docear. Assuming that Docear's users create enough mindmaps, the link-based ideas might be interesting to pursue. 
Those ideas that depend on the availability of terms seem more feasible, considering the content of mind-maps. However, only a small number of mind-maps are publicly available (around 300,000). This makes the ideas less interesting for third parties who do not offer their own mind-mapping software. The same is true for developers of mind-mapping software with only a few users. A mind-map search engine or trend analysis using for example only 50,000 mind-maps, cannot attract many people. For the major players, such as XMind, FreeMind, or MindMeister, this might be different. They potentially have access to millions of mind-maps, which should be sufficient to achieve reasonable results. One idea is also relevant for the less popular mindmapping tools, namely user modelling. User modelling, more precisely recommender system, personalized advertisement, or expert search, should be well applicable even with few users. User modelling has also the highest acceptance rate among the users. User Modelling for a recommender system was accepted by $61 \%$ or the users. User acceptance of the other ideas was lower. Around $10 \%$ of mind-maps are published, and around $30-40 \%$ of users accept IR to enhance external applications.

Table 1: Feasibility of the ideas

\begin{tabular}{|l|c|c|c|c|c|}
\cline { 2 - 5 } \multicolumn{1}{c|}{} & \multicolumn{2}{c|}{ Mind Map Availability } & \multicolumn{2}{c|}{} \\
\cline { 2 - 5 } \multicolumn{1}{c|}{} & $\begin{array}{c}\text { For 3rd } \\
\text { parties }\end{array}$ & $\begin{array}{c}\text { For MM tool } \\
\text { developers }\end{array}$ & $\begin{array}{c}\text { Content } \\
\text { Suitability }\end{array}$ & $\begin{array}{c}\text { Users ' } \\
\text { Acceptance }\end{array}$ & Overall \\
\hline Search Engine & Low & Depends & Good & Low & Low \\
\hline Document Indexing & Low & Depends & Low & Medium & Low \\
\hline Document Relatedness & Low & Depends & Low & Medium & Low \\
\hline Document Summarization & Low & Depends & Low & Medium & Low \\
\hline Impact Analysis & Low & Depends & Low & Medium & Low \\
\hline Trend Analysis & Low & Depends & Medium & Medium & Medium \\
\hline Semantic Analysis & Low & Depends & Good & Medium & Medium \\
\hline User Modeling & --- & Good & Good & Good & Good \\
\hline
\end{tabular}

Overall, user modelling seems to be the most promising idea: The content of mindmaps is suitable, user acceptance is rather high, and user modelling is relevant for all developers of mind-mapping software, and companies whose employees use mindmaps. In addition, user modelling directly benefits the mind-mapping tools and may be fundamentally important for a company. For instance, Google is generating almost its entire profit from personalized advertisements [14], and Amazon is also making a significant amount of revenue through its recommender system [15]. In contrast, applications such as semantic analysis are usually not fundamental to a company's business.

However, user modelling based on mind-maps already had been implemented, but results indicate that it is not as promising as our analysis suggests. MindMeister and Mindomo created user models for displaying personalized advertisement but both abandoned this after a while. This leads to the question, whether mind-maps actually can successfully be utilized by user modelling systems.

\section{Prototype}

To analyze whether user modelling based on mind-maps can be done effectively, we integrated a recommender system into our mind-mapping tools SciPlore MindMap- 
ping (SPLMM) [13], and its successor Docear [5]. Both tools are primarily used by researchers. Therefore, the recommender system recommends research papers. We implemented different recommendation approaches that we evaluated using clickthrough rate (CTR), i.e. the ratio of clicked recommendations against the number of displayed recommendations. Please note that due to space restriction we may only provide superficial information on the recommender system and its evaluation. We are about to publish a paper that will present the architecture of Docear's recommender system in more detail, as well as a discussion on the suitability of CTR as an evaluation metric for recommender systems. These papers will be available soon at http://www.docear.org/publications/.

For SPLMM, we implemented an approach similar to MindMeister's approach. Each time, a user modified, i.e. edited or created, a node, the terms of that node were send as search query to Google Scholar. Google Scholar's Top 3 results were shown in a separate window above the currently opened mind-map. Between July and December 2011, 78,698 recommendations were displayed, of which 221 were clicked, i.e. an overall CTR of $0.28 \%$ was achieved (Figure 8). A CTR of $0.28 \%$ is low. If MindMeister and Mindomo should have achieved similarly CTRs, it is no surprise that they abandoned the personalized advertisement.

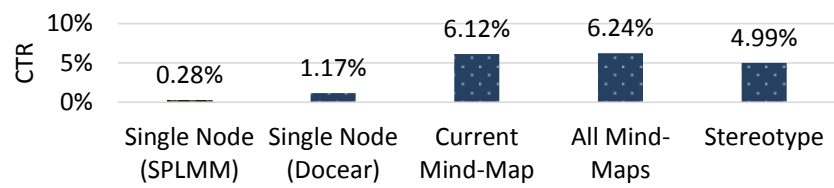

Figure 8: CTR of different approaches

In Docear, we integrated a new recommender system [4]. The new system showed recommendations only when users explicitly requested them, or automatically every five days on start-up of Docear. Recommendations were based on Docear's own document corpus, consisting of around 1.8 million full-text articles. The recommender system used four different approaches and displayed 21,445 recommendations between July 2012 and February 2013. The first approach made use of the terms of the last modified node, similar to the approach of SPLMM. This led to a CTR of $1.17 \%$ (Figure 8). The reasons why CTR was around four times higher than CTR in SPLMM, may be manifold. Maybe, the lower frequency of displaying recommendations (every five days instead of continuously) or the source (Docear's corpus vs Google Scholar), influenced CTR. However, $1.17 \%$ is still a rather low CTR. The second approach utilized the most frequent words of the user's current mind-map. This increased CTR to $6.12 \%$. When the most frequent words of all mind-maps were utilized, CTR was also above $6 \%$. For the fourth approach, we manually compiled a list of ten research articles relating to academic writing. Most of Docear's users are researchers and therefore we assumed that these articles would be relevant to most of Docear's users. When recommendations were given based on this approach - the stereotype approach [16] - CTR was 4.99\%.

The results show that a single node, typically containing two to three words, does not express a user's information needs thoroughly. Instead, entire mind-maps are needed for analysis. To analyze this factor in more detail, we modified the recommender system, so it randomly chose the number of nodes to analyze. The results show that there is a strong correlation between the number of nodes analyzed and the 
CTR (Figure 9). When the recommender system utilized only the last $1-9$ modified nodes, CTR was $3.16 \%$ on average. When 10 to 49 nodes were utilized, CTR increased to $4 \%$ on average. Utilizing between 500 and 999 nodes resulted in the highest CTR $(7.47 \%)$. When more than 1,000 nodes were utilized, CTR began to decrease (though, the difference is not statistically significant).

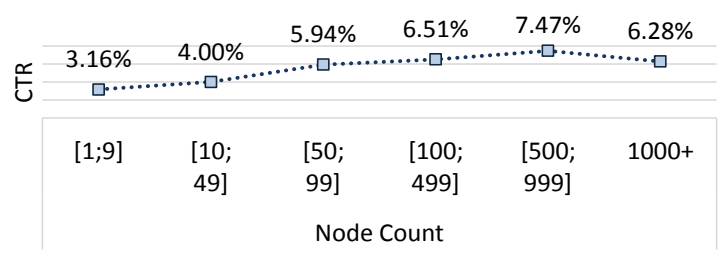

Figure 9: CTR by number of analyzed nodes

\section{Summary}

Mind-maps have thus far been widely neglected by the information retrieval community. We found that there are more than 100 mind-mapping tools and that, based on search volume, the popularity of mind-mapping is comparable to the popularity of note taking, file management, or crowdsourcing. Popular mind-mapping tools, such as XMind, are as popular as popular reference management software (e.g. Zotero), or Tech Blogs (e.g. TechCrunch). Overall, we estimated, there are around 2 million people who actively create mind-maps using a mind-mapping software. Based on these numbers, we conclude that it is worth to research whether the developers of mindmapping tools, and their users, might benefit from new applications, which utilize mind-maps.

We presented eight ideas of how mind-maps could be utilized to enhance information retrieval applications: search engines for mind-maps could help to find interesting information; user modelling based on mind-maps could enable the implementation of recommender systems, personalized advertisement, and expert search; anchor text analysis applied to mind-maps could enhance the indexing of web-pages and other documents; similarly, anchor-text analysis could enhance the summarization of web-pages and documents being linked in mind-maps; citation and link analysis could help to calculate document relatedness, which might be useful to enhance search engines or recommender systems; similarly, citation and link analysis in mind-maps could be used for impact and trend analysis; finally, semantic analyses could be applied to mind-maps to identify synonyms and other relationships of words,

Not all ideas are equally feasible. We analyzed the content of mind-maps and learned that mind-maps often do not contain any citations or links. In addition, there are only around 300,000 mind-maps publicly available, although around 5 million mind-maps are created each year. The user's acceptance to utilize their mind-maps was mediocre. $38 \%$ of the users allowed the use of their mind-maps for e.g. anchor text analysis, $61 \%$ accepted recommendations based on their mind-maps. We concluded that, out of the eight ideas, user modelling is the most feasible use case. The content of mind-maps is suitable for user modelling, the users' acceptance seems 
reasonably high, and user modelling is relevant for all developers of mind-mapping software, not only the major players.

We implemented a prototype of a user modelling system, namely a research paper recommender system, and, overall, results are promising. While the most simple user modelling approach - utilizing terms of the currently edited or created node - performed poorly (CTRs around 1\% and lower), utilizing terms of users' entire mindmaps achieved click-through rates above 6\%. This shows that user modelling based on mind-maps is not trivial, and strongly depends on the applied approaches. Further research is required to identify the unique characteristics of mind-maps, and to use these characteristics successfully in user modelling systems such as expert search, and recommender systems.

\section{References}

1. Google: Ads in Gmail and your personal data, https://support.google.com/mail/answer/6603, (2012).

2. Zubiaga, A., Martinez, R., Fresno, V.: Getting the most out of social annotations for web page classification. Proceedings of the 9th ACM symposium on Document engineering. 74-83 (2009).

3. Jacso, P.: Testing the calculation of a realistic h-index in Google Scholar, Scopus, and Web of Science for FW Lancaster. Library Trends. 56, 784-815 (2008).

4. Beel, J., Langer, S., Genzmehr, M., Nürnberger, A.: Introducing Docear's Research Paper Recommender System. Proceedings of the 13th ACM/IEEE-CS Joint Conference on Digital Libraries (JCDL'13). pp. 459-460. ACM (2013).

5. Beel, J., Gipp, B., Langer, S., Genzmehr, M.: Docear: An Academic Literature Suite for Searching, Organizing and Creating Academic Literature. Proceedings of the 11th International ACM/IEEE conference on Digital libraries. pp. 465-466. ACM (2011)

6. Nesbit, J.C., Adesope, O.O.: Learning with concept and knowledge maps: A meta-analysis. Review of Educational Research. 76, 413 (2006).

7. Brucks, C., Schommer, C.: Assembling Actor-based Mind-Maps from Text Stream. arXiv preprint (abs/0810.4616). (2008).

8. Mahler, T., Weber, M.: Dimian-Direct Manipulation and Interaction in Pen Based Mind Mapping. Proceedings of the 17th World Congress on Ergonomics, IEA 2009 (2009).

9. Beel, J., Langer, S.: An Exploratory Analysis of Mind Maps. Proceedings of the 11th ACM Symposium on Document Engineering (DocEng'11). pp. 81-84. ACM (2011).

10. Beel, J., Gipp, B., Stiller, J.-O.: Information Retrieval on Mind Maps - What could it be good for? Proceedings of the 5th International Conference on Collaborative Computing: Networking, Applications and Worksharing (CollaborateCom'09). pp. 1-4. (2009).

11. Gipp, B., Beel, J.: Citation Proximity Analysis (CPA) - A new approach for identifying related work based on Co-Citation Analysis. Proceedings of the 12th International Conference on Scientometrics and Informetrics (ISSI'09). pp. 571-575. International Society for Scientometrics and Informetrics, Rio de Janeiro (Brazil) (2009).

12. Chi, Y., Tseng, B.L., Tatemura, J.: Eigen-trend: trend analysis in the blogosphere based on singular value decompositions. Proceedings of the 15th ACM international conference on Information and knowledge management. pp. 68-77. ACM (2006).

13. Beel, J., Gipp, B., Mueller, C.: SciPlore MindMapping' - A Tool for Creating Mind Maps Combined with PDF and Reference Management. D-Lib Magazine. 15, (2009).

14. Google: Annual Report. http://investor.google.com/pdf/2010_google_annual_report.pdf. (2010).

15. Kiwitobes: Lessons on recommendation systems. Blog, http://blog.kiwitobes.com/?p=58. (2011).

16. Rich, E.: User modeling via stereotypes. Cognitive science. 3, 329-354 (1979). 